\title{
Analytical Solutions of One-Dimensional Temporally Dependent Advection-Diffusion Equation along Longitudinal Semi-Infinite Homogeneous Porous Domain for Uniform Flow
}

\author{
${ }^{1}$ Atul Kumar, ${ }^{2}$ Dilip Kumar Jaiswal, ${ }^{3}$ R. R. Yadav \\ Department of Mathematics \& Astronomy, Lucknow University, Lucknow-226007, U.P, India
}

\begin{abstract}
Analytical solutions are obtained for one-dimensional advection-diffusion equation with variable coefficients in longitudinal semi-infinite homogeneous porous medium for uniform flow. The solute dispersion parameter is considered temporally dependent while the velocity of the flow is considered uniform. The first order decay and zero-order production terms are considered inversely proportional to the dispersion coefficient. Retardation factor is also considered in present paper. Analytical solutions are obtained for two cases: former one is for uniform input point source and latter case is for increasing input point source where the solute transport is considered initially solute free. The Laplace transformation technique is used. New space and time variables are introduced to get the analytical solutions. The solutions in all possible combinations of increasing or decreasing temporally dependence dispersion are compared with each other with the help of graph. It is observed that the concentration attenuation with position and time is the fastest in case of decreasing dispersion in accelerating flow field.
\end{abstract}

Keywords: Advection, Diffusion, First-order Decay, Zero-order Production, Retardation Factor, Homogeneous Medium.

\section{Introduction}

Advection-diffusion equation describes the solute transport due to combined effect of diffusion and convection in a medium. It is a partial differential equation of parabolic type, derived on the principle of conservation of mass using Fick's law. Its analytical/numerical solutions along with an initial condition and two boundary conditions help understand the contaminant or pollutant concentration distribution behavior through an open medium like air, rivers, lakes and porous medium like aquifer, on the basis of which remedial processes to reduce or eliminate the damages may be enforced. It has wide applications in other disciplines too, like soil physics, petroleum engineering, chemical engineering and bio sciences. This equation is also used in describing similar phenomena in bio-physics and bio-medical sciences. A quantitative understanding of the transport of pollutants in groundwater is of great importance from the environmental perspective. Some environmental pollution scenarios involving groundwater contamination are very real. Effect of contamination depends on nature and levels of the toxicants, sometimes causing serious health hazards even at low level, therefore most of the times remediation becomes a necessity for achieving sustainability. If groundwater becomes polluted, it is very difficult to rehabilitate. The slow rate of groundwater flow and low microbiological activities limit any self-purification processes which takes place in days or weeks, in surface water systems can take decades. Mathematical modeling of contaminant behavior in porous media is considered to be a powerful tool for a wide range of pollution problems related with groundwater quality rehabilitation.

The development of research in the field of solute transport through a medium stems from the time the concern over the degradation of air, soil, surface water bodies and groundwater grew. Following the formulation of the advection-diffusion equation (ADE) and the theories relating dispersion and velocity ${ }^{1-4}$, the number of solute transport studies has increased considerably. Many of these studies aimed at solving the ADE for nonreactive and reactive solutes, subject to various initial and boundary conditions. A number of analytical solutions describing solute moving through one-dimensional media, considering adsorption, first-order decay and zero-order production, are compiled ${ }^{5-6}$.

Analytical solutions have obtained for uniform flow through a homogeneous and isotropic porous domain $^{7-8}$. It was done either by introducing moving coordinates ${ }^{9-14}$ or by introducing another dependent variable $^{15-16}$. Then Laplace transformation technique has been used to get desired solutions. In addition to this method, Hankel transform method, Aris moment method, perturbation approach, method using Green's function, superposition method have also been used to get the analytical solutions of the advection-diffusion equations in one, two and three dimensions. But Laplace transformation technique has been commonly used because of being simpler than other methods and the analytical solutions using this method being more reliable in verifying the numerical solutions in terms of the accuracy and the stability. The effects of initial and boundary conditions on the distribution of the tracer in time and distance for several one-dimensional systems (infinite, 
semi-infinite, and finite) of tagged liquid flowing through a solid matrix, and the effects of hydrodynamic dispersion, diffusion and radioactive decay are calculated ${ }^{17}$. An analytical solution of a cation adsorption soil problem in detail by an integral transform method including effects of axial dispersion is derived ${ }^{18}$. Two different analytic solutions are obtained to a single diffusion-convection equation over a finite domain ${ }^{19}$. The study of the dispersion of a reactive gaseous species emitted from an elevated time-dependent point source undergoing a first-order chemical reaction removal process such as rainout/washout and forming secondary species by solving unsteady-state three-dimensional diffusion equation by prescribing different forms of fluxes at the source ${ }^{20}$.

An analytical solution is studied for non equilibrium transport of reactive solutes in the unsaturated zone during an infiltration and redistribution cycle ${ }^{21}$. Similar coefficients had earlier been derived for these two problems in one spatial dimension. Solute transport with spatially and temporally varying dispersion coefficient through homogeneous porous media is studied analytically ${ }^{22}$. Pollutant dispersion in the planetary boundary layer for advection-diffusion equation studied analytically ${ }^{23}$. Dispersion of pollutants from a point source investigated analytically taking into consideration the vertical variation of both wind speed and eddy diffusivity $^{24}$. Solute transport in rivers with influence of transient storage in hyporheic zones and advectiondispersion equation for transport with a sink term in hyporheic zones described analytically for instantaneous injection of a conservative tracer in an infinite uniform river reach with steady flow ${ }^{25}$. A numerical comparison, under statistical and computational point of view, between semi-analytical Eulerian and Lagrangian dispersion models to simulated the ground-level concentration values of a passive pollutant released from a low height source. The Eulerian approach is based on the solution of the advection-diffusion equation by the Laplace transform technique ${ }^{26}$. Interaction occurs in leachate contaminated soils described for reactive solute transport model $^{27}$. Recently, analytical solutions ${ }^{28-32}$ has obtained for temporally and spatially dependent solute dispersion in one-dimensional finite and semi-infinite media. The solute dispersion parameter and velocity of the flow is considered variable coefficients along homogeneous and inhomogeneous media. Analytical solutions are obtained by using Laplace transform technique.

In the present paper, the analytical solutions of a one-dimensional advection-diffusion equation with variable coefficients are obtained. The variability of the coefficients (dispersivity of the solute transport and velocity of the flow domain) is considered in more general and reasonable form. The medium is considered semi-infinite homogeneous in longitudinal direction. Two cases are considered. First one is for uniform input point source and second is for increasing input point source. The solute dispersion parameter is considered temporally dependent while the flow velocity is uniform ${ }^{34}$. The first order decay and zero order production terms are also considered and are inversely proportional to the dispersion coefficient ${ }^{5}$. In each case the domain is initially solute free. The input condition is introduced at the origin of the domain and second condition is considered at the end of the domain. New space and time variables are introduced at the different stage through different transformations. It helps to reduce the variable coefficients into constant coefficients. So a much simpler but more viable Laplace transformation technique is used to get the analytical solutions. The solutions in all possible combinations of increasing or decreasing temporally dependence are compared with each other with the help of graph.

\section{Methodology}

Analytical solutions and field observations are used to address groundwater flow and contaminant transport problems in porous media. Analytical methods basically provide solutions to governing equations of groundwater flow and contaminant transport with simplified boundary conditions and hydro-geological properties. Limitations for analytical solutions of groundwater flow and contaminant transport in porous medium includes: assumptions of a uniform porosity, uniform hydraulic conductivity and specific yield, single inland boundary condition, and single-phase homogeneous fluid, among others of porous medium The Laplace transformation can be defined as;

If $f(x, t)$ is a any function defined in $a \leq x \leq b$ and $t>0$, then its Laplace transform with respect to $t$ is denoted by $L\{f(x, t)\}=F(x, p)$ and is defined by;

$$
L\{f(x, t)\}=F(x, p)=\int_{0}^{\infty} e^{-p t} f(x, t) d t, \quad p>0
$$

where $p$ is called the transform variable, which is a complex variable.

The inverse Laplace transform is denoted by $L^{-1}\{F(x, p)\}=f(x, t)$ and defined by the complex variable;

$$
L^{-1}\{F(x, p)\}=f(x, t)=\frac{1}{2 \pi i} \int_{c-i \infty}^{c+i \infty} e^{-p t} F(x, p) d p, c>0
$$




\section{Advection-Diffusion Equation}

In one space dimension the linear advection-diffusion equation may be written as

$$
\frac{\partial c}{\partial t}+\frac{1-n}{n} \frac{\partial F}{\partial t}=\frac{\partial}{\partial x}\left(D(x, t) \frac{\partial c}{\partial x}-u(x, t) c\right)-\gamma(x, t) c+\mu(x, t)
$$

where $c$ is the solute concentration at position $x$ at time $t$ in liquid phase and $F$ is the solute concentration in solid phase, $n$ is the porosity of the medium. $D(x, t)$ is represent the solute dispersion parameter and is called the dispersion coefficient if it is uniform and steady, $u(x, t)$ is the flow velocity which satisfies the Darcy's law. $\gamma(x, t)$ is the first-order decay and $\mu(x, t)$ is the zero-order production which represents internal/external production of the solute. In subsurface, solute transport processes affect all contaminants at equal rates, while the presence of retardation, first-order decay and zero order production may influence various contaminants at various rates. If contaminants passes through an aquifer or groundwater, their movement rate may be less than the average groundwater flow rate.

Considered two cases, as follows

$$
F=K_{1} c+K_{2}
$$

and

$$
\frac{\partial F}{\partial t}=K_{1} c-K_{2} F
$$

respectively, equilibrium and non-equilibrium relationship between the concentrations in the two phases. For simplicity, the former relationship is adopted in the present analysis ${ }^{34}$. Using Eq. (4) in Eq. (3) one may obtains,

$$
\begin{aligned}
& \frac{\partial c}{\partial t}+\frac{1-n}{n} \frac{\partial\left(K_{1} c+K_{2}\right)}{\partial t}=\frac{\partial}{\partial x}\left(D(x, t) \frac{\partial c}{\partial x}-u(x, t) c\right)-\gamma(x, t) c+\mu(x, t) \\
& R_{d} \frac{\partial c}{\partial t}=\frac{\partial}{\partial x}\left(D(x, t) \frac{\partial c}{\partial x}-u(x, t) c\right)-\gamma(x, t) c+\mu(x, t)
\end{aligned}
$$

where $R_{d}=\left(1+\frac{1-n}{n} K_{1}\right)$ is retardation factor.

\section{Analytical Solutions}

Let us write $D(x, t), u(x, t), \gamma(x, t)$ and $\mu(x, t)$ in Eq. (3) as

$$
D(x, t)=D_{0} f_{1}(x, t) ; \quad u(x, t)=u_{0} f_{2}(x, t)
$$

and the first order decay, $\gamma(x, t)$ and zero order production, $\mu(x, t)$ terms which is inversely proportional to the dispersion coefficient i.e.

$$
\gamma(x, t)=\gamma_{0} / f_{1}(x, t) ; \mu(x, t)=\mu_{0} / f_{1}(x, t)
$$

respectively, where $D_{0}\left(L^{2} T^{-1}\right), u_{0}\left(L T^{-1}\right), \gamma_{0}\left(T^{-1}\right)$ and $\mu_{0}\left(M L^{-3} T^{-1}\right)$ are constants. Eq. (7) is rewritten as

$$
R_{d} \frac{\partial c}{\partial t}=\frac{\partial}{\partial x}\left(D_{0} f_{1}(x, t) \frac{\partial c}{\partial x}-u_{0} f_{2}(x, t) c\right)-\gamma_{0} c / f_{1}(x, t)+\mu_{0} / f_{1}(x, t)
$$

Let us introduce a new independent variable, $X$ by a transformation ${ }^{28,31}$

$$
X=\int \frac{d x}{f_{1}(x, t)} \quad \text { or } \quad \frac{d X}{d x}=\frac{1}{f_{1}(x, t)}
$$

Applying the transformation of Eq. (11) on the partial differential equation, Eq. (10) becomes

$$
f_{1}(x, t) R_{d} \frac{\partial c}{\partial t}=D_{0} \frac{\partial^{2} c}{\partial X^{2}}-u_{0} \frac{\partial}{\partial X}\left\{f_{2}(x, t) c\right\}-\gamma_{0} c+\mu_{0}
$$

Now this partial differential equation is solved analytically using the initial and boundary conditions of temporally dependent dispersion along a uniform flow.

\section{IV.I Temporally Dependent Dispersion Along Uniform Flow}

The solute dispersion parameter is supposed to be time dependent. So in Eq. (8) we consider

$$
f_{1}(x, t)=f(m t) \text { and } f_{2}(x, t)=1
$$


where $m\left(T^{-1}\right)$ is a unsteady parameter whose dimension is inverse of that of the time variable $t . f(m t)$ is chosen such that for $m=0$ or $t=0, f(m t)=1$. Thus $f(m t)$ is an expression in the non-dimensional variable $m t . m=0$ corresponds to the temporally independent dispersion. In view of the expressions in Eq. (13), the constants $D_{0}$ and $u_{0}$ in Eq. (8) may be referred to as the initial dispersion coefficient $\left(L^{2} T^{-1}\right)$ and the uniform velocity $\left(L T^{-1}\right)$, respectively. Further from Eq. (11) we have

$$
X=\int \frac{d x}{f(m t)} \quad \text { or } \quad \frac{d X}{d x}=\frac{1}{f(m t)}
$$

The partial differential equation Eq. (12) will become

$$
f(m t) R_{d} \frac{\partial c}{\partial t}=D_{0} \frac{\partial^{2} c}{\partial X^{2}}-u_{0} \frac{\partial c}{\partial X}-\gamma_{0} c+\mu_{0}
$$

Further another independent variable, $T$ is introduced using the transformation ${ }^{35}$

$$
T=\int_{0}^{t} \frac{d t}{f(m t)}
$$

As a result, Eq. (16) reduces to a partial differential equation with constant coefficients, we have

$$
R_{d} \frac{\partial c}{\partial T}=D_{0} \frac{\partial^{2} c}{\partial X^{2}}-u_{0} \frac{\partial c}{\partial X}-\gamma_{0} c+\mu_{0}
$$

The dimension of variable $X$ defined by Eq. (14) and that of variable $T$ defined by Eq. (16) remain those of $x$ and $t$, respectively and are referred to as the new space variable and the new time variable, respectively. The new time variable obtained from Eq. (16) satisfies the conditions $T=0$ for $t=0$ and $T=t$ for $m=0$. The first condition ensures that the nature of the initial condition does not change in the new time variable domain. The second condition refers to the temporally independent dispersion and Eq. (10) reduces to one with constant coefficients

\section{IV.I.I Uniform Input Point Source Condition}

To proceed further, initial and boundary conditions are defined. The semi-infinite medium is supposed. In case of uniform input point source, initially the region of interest is free from the pollution at the beginning of the source is assumed. Thus the initial condition is

$$
c(x, t)=0 ; t=0, x \geq 0
$$

Further the continuous input concentration is introduced at the origin of the domain. The second boundary condition is of flux type of homogeneous nature at the infinity. Thus the two boundary conditions are as follows

$$
\begin{aligned}
& c(x, t)=C_{0} ; x=0, t>0, \\
& \frac{\partial c(x, t)}{\partial x}=0 ; x \rightarrow \infty, t \geq 0 .
\end{aligned}
$$

The initial and boundary conditions (18-20) may be written in terms of new independent variables as

$$
\begin{aligned}
& c(X, T)=0 ; T=0, X \geq 0 \\
& c(X, T)=C_{0} ; X=0, T>0, \\
& \frac{\partial c(X, T)}{\partial X}=0 ; X \rightarrow \infty, T \geq 0 .
\end{aligned}
$$

Now Laplace transform which are defined in Eq. (1) may be used to get the analytical solution. Using the following transformation (24) to eliminate the convection term, first order decay and zero order production from advection-diffusion equation Eq. (17) as follows

$$
c(X, T)=K(X, T) \exp \left\{\frac{u_{0}}{2 D_{0}} X-\frac{1}{R_{d}}\left(\frac{u_{0}^{2}}{4 D_{0}}+\gamma_{0}\right) T\right\}+\frac{\mu_{0}}{\gamma_{0}}
$$

We get the initial and boundary value problem in terms of new dependent variable $K(X, T)$ as

$$
\begin{aligned}
& R_{d} \frac{\partial K}{\partial T}=D_{0} \frac{\partial^{2} K}{\partial X^{2}} \\
& K(X, T)=-\frac{\mu_{0}}{\gamma_{0}} \exp \left\{-\frac{u_{0}}{2 D_{0}} X\right\} ; T=0, X \geq 0
\end{aligned}
$$




$$
\begin{aligned}
& K(X, T)=\left(C_{0}-\frac{\mu_{0}}{\gamma_{0}}\right) \exp \left\{\alpha^{2} T\right\} ; X=0, T>0, \\
& \frac{\partial K}{\partial X}+\frac{u_{0}}{2 D_{0}} K=0 ; X \rightarrow \infty, T \geq 0 .
\end{aligned}
$$

where $\alpha^{2}=\frac{1}{R_{d}}\left(\frac{u_{0}^{2}}{4 D_{0}}+\gamma_{0}\right)$.

Applying the Laplace transformation in set of above initial and boundary value problem reduces to an ordinary differential equation of second order boundary value problem, which comprises of following three equations

$$
\frac{d^{2} \bar{K}}{d X^{2}}-\frac{p R_{d}}{D_{0}} \bar{K}=\frac{\mu_{0} R_{d}}{\gamma_{0} D_{0}} \exp \left\{-\frac{u_{0}}{2 D_{0}} X\right\}
$$

and boundary conditions are

$$
\begin{aligned}
& \bar{K}(X, p)=\left(C_{0}-\frac{\mu_{0}}{\gamma_{0}}\right) \frac{1}{\left(p-\alpha^{2}\right)} ; X=0, \\
& \frac{d \bar{K}}{d X}+\frac{u_{0}}{2 D_{0}} \bar{K}=0 ; X \rightarrow \infty .
\end{aligned}
$$

where $p$ is Laplace parameter is defined in Eq. (1).

Thus, the general solution of ordinary differential equation (29) may be written as

$$
\bar{K}(X, p)=c_{1} \exp \left\{-\sqrt{\frac{p R_{d}}{D_{0}}} X\right\}+c_{2} \exp \left\{\sqrt{\frac{p R_{d}}{D_{0}}} X\right\}+\frac{\mu_{0}}{\gamma_{0}\left(p-\beta^{2}\right)} \exp \left\{-\frac{u_{0}}{2 D_{0}} X\right\}
$$

where $\beta^{2}=\frac{u_{0}^{2}}{4 R_{d} D_{0}}$.

Now, using boundary conditions Eq. (30) and Eq. (31) in general solution Eq. (32) for eliminating the arbitrary constant $c_{1}$ and $c_{2}$, we get

$$
\begin{aligned}
& c_{1}=\left(C_{0}-\frac{\mu_{0}}{\gamma_{0}}\right) \frac{1}{\left(p-\alpha^{2}\right)}-\frac{\mu_{0}}{\gamma_{0}} \frac{1}{\left(p-\beta^{2}\right)} \text { at } X=0 \\
& c_{2}=0 \text { at } X \rightarrow \infty
\end{aligned}
$$

Thus, the particular solution in the Laplace domain may be written as

$$
\begin{aligned}
\bar{K}(X, p)=\left[\left(C_{0}-\frac{\mu_{0}}{\gamma_{0}}\right) \frac{1}{\left(p-\alpha^{2}\right)}-\frac{\mu_{0}}{\gamma_{0}}\right. & \left.\frac{1}{\left(p-\beta^{2}\right)}\right] \exp \left\{-\sqrt{\frac{p R_{d}}{D_{0}}} X\right\} \\
& +\frac{\mu_{0}}{\gamma_{0}} \exp \left\{-\frac{u_{0}}{2 D_{0}} X\right\} \frac{1}{\left(p-\beta^{2}\right)}
\end{aligned}
$$

Applying inverse Laplace transform, the analytical solution of advection-diffusion equation for uniform input point source for continuous nature may be written in terms of $c(x, T)$ by using back transformations Eq. (24), Eq. (16) and Eq. (14) as

$$
c(x, T)=\frac{\mu_{0}}{\gamma_{0}}+\left(C_{0}-\frac{\mu_{0}}{\gamma_{0}}\right) F_{1}(x, T)-\frac{\mu_{0}}{\gamma_{0}} F_{2}(x, T)
$$

where

$$
\begin{array}{r}
F_{1}(x, T)=\frac{1}{2} \exp \left[\left(\frac{\left\{u_{0}-\left(u_{0}^{2}+4 \gamma_{0} D_{0}\right)^{1 / 2}\right\}}{2 D_{0}}\right) \frac{x}{f(m t)}\right] \\
\times \operatorname{erfc}\left[\frac{\left\{R_{d} x / f(m t)-\left(u_{0}^{2}+4 \gamma_{0} D_{0}\right)^{1 / 2} T\right\}}{2 \sqrt{D_{0} R_{d} T}}\right]
\end{array}
$$




$$
\begin{array}{r}
+\frac{1}{2} \exp \left[\left(\frac{\left\{u_{0}+\left(u_{0}^{2}+4 \gamma_{0} D_{0}\right)^{1 / 2}\right\}}{2 D_{0}}\right) \frac{x}{f(m t)}\right] \\
\times \operatorname{erfc}\left[\frac{\left\{R_{d} x / f(m t)-\left(u_{0}^{2}+4 \gamma_{0} D_{0}\right)^{1 / 2} T\right\}}{2 \sqrt{D_{0} R_{d} T}}\right], \\
F_{2}(x, T)=\exp \left\{-\frac{\gamma_{0} T}{R_{d}}\right\}\left[1-\frac{1}{2} \operatorname{erfc}\left\{\frac{R_{d} x / f(m t)-u_{0} T}{\left.2 \sqrt{D_{0} R_{d} T}\right\}}\right.\right. \\
-\frac{1}{2} \exp \left\{\frac{u_{0}}{D_{0}} X\right\} \operatorname{erfc}\left\{\frac{R_{d} x / f(m t)+u_{0} T}{\left.\left.2 \sqrt{D_{0} R_{d} T}\right\}\right] \text { and }}\right. \\
T=\int_{0}^{t} \frac{d t}{f(m t)} \cdot
\end{array}
$$

\section{IV.I.II Increasing Input Point Source Condition}

The source of input concentration may increase with time due to variety of reasons. This type of situation may be described by a mixed type or third type condition written as follows:

$$
-D(x, t) \frac{\partial c}{\partial x}+u(x, t) c=u_{0} C_{0} \quad \text { at } \quad x=0, t>0
$$

Using Eq. (8), Eq. (13), Eq. (14) and Eq. (16) the above condition may be written in $(X, T)$ domain as

$$
-D_{0} \frac{\partial c}{\partial X}+u_{0} c=u_{0} C_{0} \quad \text { at } \quad X=0, T>0
$$

Now, Eq. (36) reduces by the applying the transformation Eq. (24), into

$$
-D_{0} \frac{\partial K}{\partial X}+\frac{u_{0}}{2} K=u_{0}\left(C_{0}-\frac{\mu_{0}}{\gamma_{0}}\right) \exp \left(\alpha^{2} T\right) ; X=0, T>0, \alpha^{2}=\frac{1}{R_{d}}\left(\frac{u_{0}^{2}}{4 D_{0}}+\gamma_{0}\right)
$$

Applying the Laplace transform on Eq. (37), we have

$$
-D_{0} \frac{d \bar{K}}{d X}+\frac{u_{0}}{2} \bar{K}=u_{0}\left(C_{0}-\frac{\mu_{0}}{\gamma_{0}}\right) \frac{1}{\left(p-\alpha^{2}\right)} ; X=0
$$

Now using the condition Eq. (38) in place of Eq. (30), for eliminating the arbitrary constants $c_{1}$ and $c_{2}$ in Eq. (32), the particular solution of Eq. (29) satisfying the conditions in Eqs. (31) and (38) may be obtained as

$$
\begin{aligned}
& c_{1}=\frac{u_{0}}{\sqrt{R_{d} D_{0}}}\left(C_{0}-\frac{\mu_{0}}{\gamma_{0}}\right) \frac{1}{\left(p-\alpha^{2}\right)(\sqrt{p}+\beta)}-\frac{u_{0}}{\sqrt{R_{d} D_{0}}} \frac{\mu_{0}}{\gamma_{0}} \frac{1}{\left(p-\beta^{2}\right)(\sqrt{p}+\beta)} \text { at } X=0 \\
& c_{2}=0 \text { at } X \rightarrow \infty
\end{aligned}
$$

Thus, the particular solution in the Laplace domain may be written as

$$
\begin{gathered}
\bar{K}(X, p)=\frac{u_{0}}{\sqrt{R_{d} D_{0}}}\left(C_{0}-\frac{\mu_{0}}{\gamma_{0}}\right) \frac{\exp \left\{-X \sqrt{p R_{d} / D_{0}}\right\}}{\left(p-\alpha^{2}\right)(\sqrt{p}+\beta)}-\frac{u_{0}}{\sqrt{R_{d} D_{0}}} \frac{\mu_{0}}{\gamma_{0}} \frac{\exp \left\{-X \sqrt{p R_{d} / D_{0}}\right\}}{\left(p-\beta^{2}\right)(\sqrt{p}+\beta)} \\
+\frac{\mu_{0}}{\gamma_{0}} \frac{\exp \left\{-\frac{u_{0}}{2 D_{0}} X\right\}}{\left(p-\beta^{2}\right)}
\end{gathered}
$$

where $\beta^{2}=\frac{u_{0}^{2}}{4 R_{d} D_{0}}, \alpha^{2}=\frac{1}{R_{d}}\left(\frac{u_{0}^{2}}{4 D_{0}}+\gamma_{0}\right)$. 
Now, applying inverse Laplace transform, the analytical solution of advection-diffusion equation for input point source for increasing nature may be written in terms of $c(x, T)$ by using back transformations Eq. (24), Eq. (16) and Eq. (14) as

$$
c(x, T)=\frac{\mu_{0}}{\gamma_{0}}+\left(C_{0}-\frac{\mu_{0}}{\gamma_{0}}\right) F_{1}(x, T)-\frac{\mu_{0}}{\gamma_{0}} F_{2}(x, T)
$$

where

$$
\begin{aligned}
& F_{1}(x, T)=\frac{u_{0}}{\left\{u_{0}+\left(u_{0}^{2}+4 \gamma_{0} D_{0}\right)^{1 / 2}\right\}} \exp \left[\left(\frac{\left\{u_{0}-\left(u_{0}^{2}+4 \gamma_{0} D_{0}\right)^{1 / 2}\right\}}{2 D_{0}}\right) \frac{x}{f(m t)}\right] \\
& \times \operatorname{erfc}\left[\frac{\left\{R_{d} x / f(m t)-\left(u_{0}^{2}+4 \gamma_{0} D_{0}\right)^{1 / 2} T\right\}}{2 \sqrt{D_{0} R_{d} T}}\right] \\
& +\frac{u_{0}}{\left\{u_{0}-\left(u_{0}^{2}+4 \gamma_{0} D_{0}\right)^{1 / 2}\right\}} \exp \left[\left(\frac{\left\{u_{0}+\left(u_{0}^{2}+4 \gamma_{0} D_{0}\right)^{1 / 2}\right\}}{2 D_{0}}\right) \frac{x}{f(m t)}\right] \\
& \times \operatorname{erfc}\left[\frac{\left\{R_{d} x / f(m t)+\left(u_{0}^{2}+4 \gamma_{0} D_{0}\right)^{1 / 2} T\right\}}{2 \sqrt{D_{0} R_{d} T}}\right] \\
& +\frac{u_{0}^{2}}{2 \gamma_{0} D_{0}} \exp \left(\frac{u_{0}}{D_{0}} \frac{x}{f(m t)}-\frac{\gamma_{0}}{R_{d}} T\right) \operatorname{erfc}\left[\frac{\left\{R_{d} x / f(m t)+u_{0} T\right\}}{2 \sqrt{D_{0} R_{d} T}}\right], \\
& F_{2}(x, T)=\exp \left\{-\frac{\gamma_{0} T}{R_{d}}\right\}\left[1-\frac{1}{2} \operatorname{erfc}\left\{\frac{R_{d} x / f(m t)-u_{0} T}{2 \sqrt{D_{0} R_{d} T}}\right\}\right. \\
& -\left(\frac{u_{0}^{2}}{\pi R_{d} D_{0}} T\right)^{1 / 2} \exp \left\{-\frac{\left(R_{d} x / f(m t)-u_{0} T\right)^{2}}{4 D_{0} R_{0} T}\right\} \\
& \left.+\frac{1}{2}\left(1+\frac{u_{0} x / f(m t)}{D_{0}}+\frac{u_{0}^{2}}{D_{0} R_{0}} T\right) \exp \left\{\frac{u_{0} x / f(m t)}{D_{0}}\right\} \operatorname{erfc}\left\{\frac{R_{d} x / f(m t)+u_{0} T}{2 \sqrt{D_{0} R_{d} T}}\right\}\right]
\end{aligned}
$$

and $T=\int_{0}^{t} \frac{d t}{f(m t)}$

\section{Illustrations And Discussion}

Concentration values are evaluated from the Eq. (34) and Eq. (40) in a finite domain $0 \leq x$ (meter) $\leq 10$ at different values of time. The input data in both the cases are chosen as $C_{0}=1.0$, initial velocity $u_{0}=0.21$ (meter/day), initial dispersion coefficient, $D_{0}=0.71$ (meter ${ }^{2} /$ day) and unsteady parameter $m=0.1$ (day) $^{-1}$. In addition to these, first order decay term $\gamma_{0}=0.02$ and zero order production term $\mu_{0}=0.0011$ are considered.

In both the cases analytical solutions may be obtained from Eqs. (34) and (40) for $f(m t)=\exp (m t)$, at different time $t$ (day) $=1.5,2.5,3.5$ and 4.5 . Thus the time interval is considered to be 1.0 day. The full line curves in all figures are drawn for exponentially increasing function of $f(m t)$ which is the unsteady pattern of the flow field nature. It may be observed that concentration values decreases with position and increases with time. In uniform input point source, concentration distribution is uniform at $x=0$, at every time $t$ and its increases with time and decreases with position, while in increasing input point source, it is not uniform at $x=0$, at every time $t$. Its increases with increasing time. 
$2.5,3.5$ and 4.5 , at $R_{d}=1.15$ and $m=0.1$ (day) $)^{-1}$ for uniform and increasing input source respectively. It may be observed that the concentration values are increases with time and decreases with position. It is also compared with the another retardation factor $R_{d}=1.35$ at time $t$ (day) $=1.5$, and another unsteady parameter $m=0.5(\text { day })^{-1}$ at time $t($ day $)=1.5$. It is observed that the solute concentration distribution is slower for the higher retardation factor from lower retardation factor and faster for the higher unsteady parameter from the lower unsteady parameter at particular times, which are shows with solid, dashed and dotted curves in Figs 1 and 4.

The concentration distribution behavior at particular time $t$ (day) $=2.5$ for three expressions (i) exponentially increasing function; $f(m t)=\exp (m t)$, (ii) exponentially decreasing function; $f(m t)=\exp (-m t)$, and (iii) sinusoidal nature; $f(m t)=1-\sin (m t)$, at one retardation factor and unsteady parameter for uniform and increasing input source respectively are shown in Figs. 2 and 5. It may be observed that solute concentrations values are higher for exponentially increasing and lower for exponentially decreasing functions and sinusoidal nature at particular position and time. It is also observed that the concentration values are higher for exponentially decreasing function from sinusoidal nature at a particular time and position.

Figs. 3 and 6 , represents the concentration distribution behavior for different time interval $t($ day $)=1.5$ to 4.5 for the three expressions (i) exponentially increasing function; $f(m t)=\exp (m t)$, (ii) exponentially decreasing function; $f(m t)=\exp (-m t)$, and (iii) sinusoidal nature; $f(m t)=1-\sin (m t)$, respectively, at a particular position $x=5.0$ (meter) for uniform and increasing input source respectively. It is observed that the solute concentration is higher in exponentially increasing function from the exponentially decreasing function and sinusoidal function at particular position.

Fig. 7 shows the effect in concentration distribution within the domain when zero-order production term is presence and absence in the domain. Comparison of concentration level is illustrated for various zeroorder production (i.e. presence and absence of production term) with fixed first order decay, unsteady parameter and retardation factor at one time for the function $f(m t)=\exp (m t)$. It is observed that, when the zero order production increases the concentration level is increase at particular time throughout domain. For better accuracy, zero order production and first order decay cannot be neglected. The nature of the function $f(m t)$ must be same has discussed in section 4.1.

Some examples of transport phenomena are smoke coming out from a chimney of a factory; particulate particles coming out of a volcano; the sewage outlet of a municipal area or effluent outlet of a factory or industry in a surface water medium; infiltrations of wastes from garbage disposal sites, septic tanks, mines, discharge from surface water bodies polluted due to industrial and municipal influents, and reaching the ground water level, particularly with rainwater.

\section{Conclusion}

Analytical solutions are obtained for temporally dependent solute dispersion for uniform and increasing input source in a semi-infinite one-dimensional longitudinal domain. Certain transformations are helped us to obtain the analytical solutions using the Laplace transform technique. LTT is simpler, more viable and commonly used in assessing the stability of numerical solutions in more realistic dispersion problems, to understand and manage the pollution distributions along ground water, surface water and air flow domains. The solutions in the both cases are obtained in terms of a function $f(\mathrm{mt})$ in time variable and may be used for a variety of time-dependent expressions. It is observed that the solute transport along the medium for an expression of $f(m t)$ of an increasing nature is faster than that obtained for $f(m t)$ of a decreasing nature and sinusoidal nature. The analytical solutions are obtained in the present work for uniform and increasing input conditions which may be useful in examining the degradation levels of the surface. Such solutions will be very useful in validating a numerical solution of a more general dispersion problem by infinite element technique ${ }^{36-37}$. The solutions in all possible combinations of increasing or decreasing temporally dependence are compared with each other with the help of graph.

\section{Acknowledgment}

This work is carried out under Post Doctoral Fellowship programme of first two authors. Financial assistance provided by the funding agency to first two authors in the form of Dr. D. S. Kothari Post Doctoral Fellowship, University Grants Commission, Government of India, are gratefully acknowledged. 


\section{References}

[1] G I Taylor, Dispersion of soluble matter in solvent flowing slowly through a tube, Proceedings of Royal Society of London, A219, 1953, 186-203.

[2] A E Scheidegger, The Physics of Flow through Porous Media (University of Toronto Press, 1957).

[3] R R Rumer, Longitudinal dispersion in steady and unsteady flow, Journal of Hydraulic Division, 88, 1962, 147-173.

[4] R A Freeze and J A Cherry, Groundwater (Prentice-Hall, New Jersey, 1979).

[5] M Th van Genuchten and W J Alves, Analytical solutions of the one-dimensional convective-dispersive solute transport equation (Technical Bulletin No 1661, US Department of Agriculture, 1982).

[6] F T Lindstrom and L Boersma, Analytical solutions for convective-dispersive transport in confined aquifers with different initial and boundary conditions, Water Resources Research, 25, 1989, 241-256.

[7] A Ogata, Theory of dispersion in granular media, US Geol. Sur. Prof. Paper 411-I, 34, 1970.

[8] M Marino, Flow against dispersion in non-adsorbing porous media, Journal of Hydrology, 37, 1978, $149-158$.

[9] A Ogata and R B Bank, A solution of differential equation of longitudinal dispersion in porous media, U. S. Geol. Surv. Prof. Pap. 411, A1-A7, 1961.

[10] D R F Harleman and R R Rumer, Longitudinal and lateral dispersion in an isotropic porous medium, Journal of Fluid Mechanics, $16(3), 1963,385-394$.

[11] V Guvanasen and R E Volker, Experimental investigations of unconfined aquifer pollution from recharge basins, Water Resources Research, 19(3), 1983, 707-717.

[12] T J Marshal, J W Holmes and C W Rose, Soil Physics (Cambridge University Press, 3rd Ed, 1996).

[13] J Bear, Dynamics of fluids in porous media (New York: Amr. Elsev. Co., 1972).

[14] M M Aral and B Liao, Analytical solutions for two-dimensional transport equation with time-dependent dispersion coefficients, Journal of Hydrologic Engineering, 1(1), 1996, 20-32.

[15] M A Marino, Longitudinal dispersion in saturated porous media, Journal of Hydraulic Division, 100, 1974, $151-157$.

[16] A N S Al-Niami and K R Rushton, Analysis of flow against dispersion in porous media, Journal of Hydrology, 33, $1977,87-97$.

[17] N D Gershon and A Nir, Effects of boundary conditions of models on tracer distribution in flow through porous mediums, Water Resources Research, 5(4), 1969, 830-839.

[18] R W Cleary and D D Adrian, Analytical solution of the convective-dispersive equation for cation adsorption in soil, Soil Sci. Soc. Am. Proc., 37, 1973, 197-199.

[19] G B Davis, A Laplace transform technique for the analytical solution of a diffusion-convection equation over a finite domain, Applied Mathematical Modeling, 9, 1985, 69-71.

[20] R S Chauhan and J B Shukla, Unsteady-state dispersion of a reactive gaseous species from an elevated time-dependent point source forming secondary species, Applied Mathematical Modeling, 13, 1989, 632-640.

[21] G Severino and P Indelman, Analytical solutions for reactive transport under an infiltration-redistribution cycle, Journal of Contaminant. Hydrology, 70, 2004, 89-115.

[22] N Su, G C Sander, F Liu, V Anh and D A Barry, Similarity solutions for solute transport in fractal porous media using a time- and scale-dependent dispersivity, Applied Mathematical Modeling, 29, 2005, 852-870.

[23] S Wortmanna, M T Vilhenaa, D M Moreira and D Buske, A new analytical approach to simulate the pollutant dispersion in the PBL, Atmospheric Environment, 39, 2005, 2171-2178.

[24] K S M Essa, S M Etman and M Embaby, New analytical solution of the dispersion equation, Atmospheric Research, 84, 2007, 337344.

[25] F D Smedt, Analytical solution and analysis of solute transport in rivers affected by diffusive transfer in the hyporheic zone, Journal of Hydrology, 339, 2007, 29-38.

[26] J C Carvalho, M T Vilhena and D M Moreira, Comparison between Eulerian and Lagrangian semi-analytical models to simulate the pollutant dispersion in the PBL, Applied Mathematical Modeling, 3, 2007, 120-129.

[27] N Singhal and J Islam, One-dimensional model for biogeochemical interactions and permeability reduction in soils during leachate permeation, Journal of Contaminant Hydrology, 96, 2008, 32-47.

[28] D K Jaiswal, A Kumar, N Kumar and R R Yadav, Analytical solutions for temporally and spatially dependent solute dispersion of pulse type input concentration in one-dimensional semi-infinite media, Journal of Hydro-environment Research, 2, 2009, 254-263.

[29] D K Jaiswal, A Kumar and R R Yadav, Analytical solution to the one-dimensional advection-diffusion equation with temporally dependent coefficients, Journal of Water Resource and Protection, 3, 2011, 76-84.

[30] A Kumar, D K Jaiswal and N Kumar, Analytical solutions of one - dimensional advection - diffusion equation with variable coefficients in a finite domain, Journal of Earth System Sciences, 118(5), 2009, 539-549.

[31] A Kumar, D K Jaiswal and N Kumar, Analytical solutions to one - dimensional advection - diffusion equation with variable coefficients in semi-infinite media, Journal of Hydrology, 380, 2010, 330-337.

[32] R R Yadav, D K Jaiswal, H K Yadav and Gulrana, One-dimensional temporally dependent advection-dispersion equation in porous Media: analytical solution, Natural Resource Modeling, 23(4), 2010, 521-539.

[33] G Matheron and G deMarsily, Is transport in porous media always diffusive? 527 A counter example, Water Resources Research, $16,1980,901-917$.

[34] L Lapidus and N R Amundson, Mathematics of adsorption in beds, VI. The effects of longitudinal diffusion in ion-exchange and chroma to graphic columns, Journal of Physical Chemistry, 56, 1952, 984-988.

[35] J Crank, The Mathematics of Diffusion (Oxford Univ. Press, London, $2^{\text {nd }}$ Ed, 1975).

[36] C Zhao and S Valliappan, Transient infinite element for contaminant transport problems, International Journal of Numerical Methods in Engineering, 37, 1994, 1143-1158.

[37] C Zhao, Dynamic and Transient Infinite Elements: Geological, Geotechnical and Geo environmental Applications (Springer, 2009). 


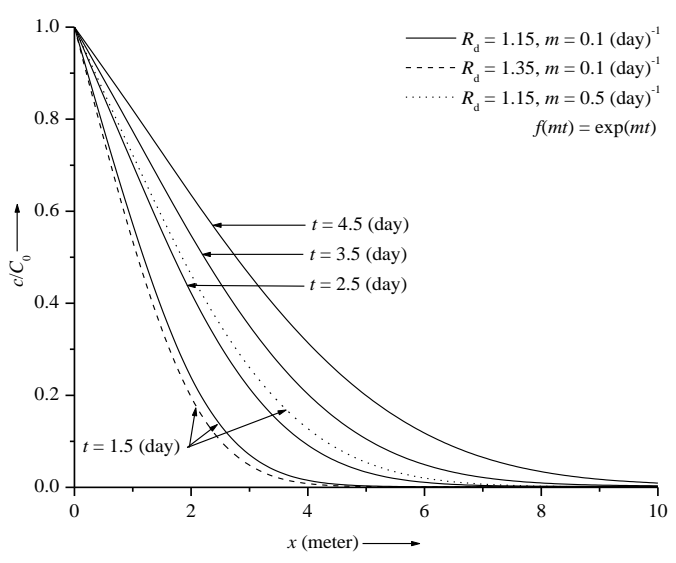

Figure 1 Distribution of the solute concentration for solution (34) at different time $t$ (day) $=1.5,2.5$, 3.5 and 4.5 , represented by four solid curves for $f(m t)=\exp (m t)$ and compare with the another retardation factor (dashed curve) and another unsteady parameter (dotted curve) at one time $t=1.5$ (day) .

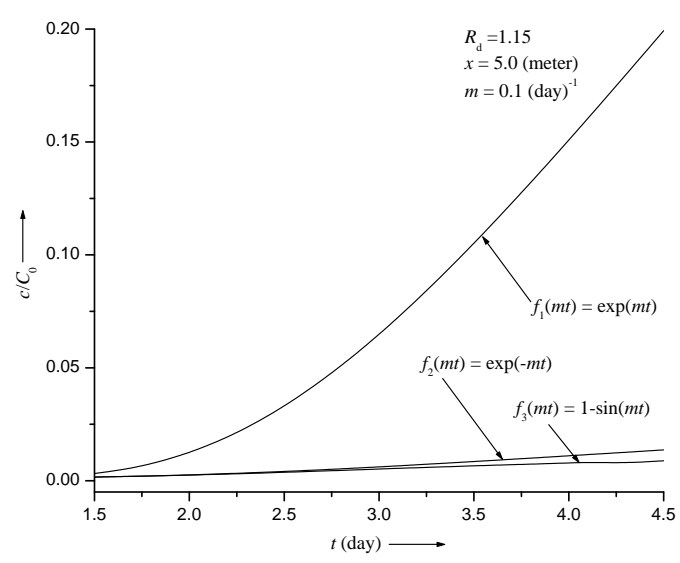

Figure 5. Comparison of the solute concentration for solution (40) at time $t$ (day) $=2.5$, represented by solid curve for $f(m t)=\exp (m t)$, dashed curve for $f(m t)=\exp (-m t)$ and dotted curve for $f(m t)=1-\sin (m t)$ at one retardation factor and unsteady parameter.

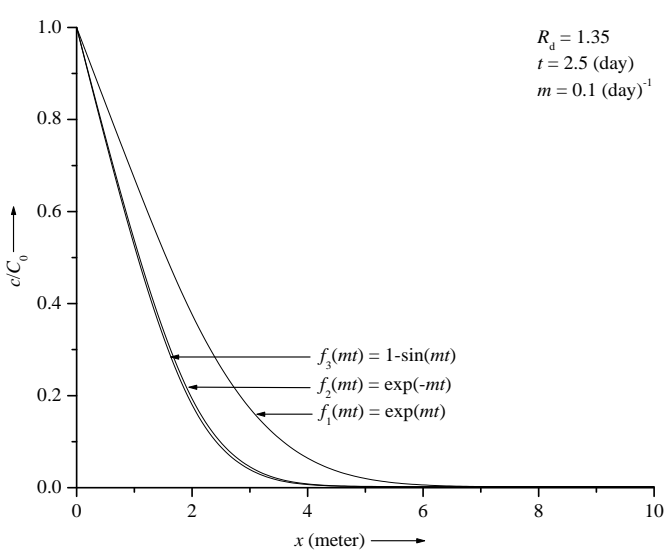

Figure 2. Comparison of the solute concentration for solution (34) at time $t($ day $)=2.5$, represented by solid curve for $f(m t)=\exp (m t)$, dashed curve for $f(m t)=\exp (-m t)$ and dotted curve for $f(m t)=1-\sin (m t)$ at one retardation factor and unsteady parameter.

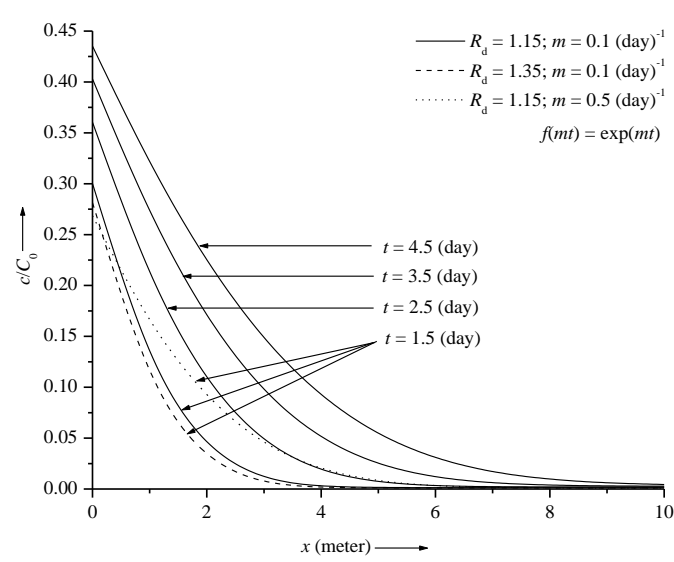

Figure 4 Distribution of the solute concentration for solution (40) at different time $t$ (day) $=1.5,2.5$, 3.5 and 4.5 , represented by four solid curves for $f(m t)=\exp (m t)$ and compare with the another retardation factor (dashed curve) and another unsteady parameter (dotted curve) at one time $t=1.5$ (day) . 


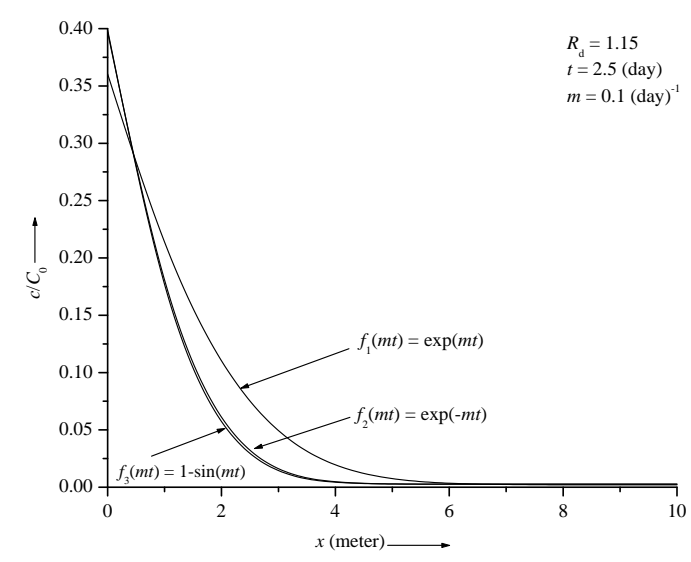

Figure 3. Comparison of the solute concentration for solution (34) at different time $t$ (day) $=1.5$ to 4.5 , represented by solid curve for $f(m t)=\exp (m t)$, dashed curve for $f(m t)=\exp (-m t)$ and dotted curve for $f(m t)=1-\sin (m t)$ at particular position $x($ meter $)=5.0$.

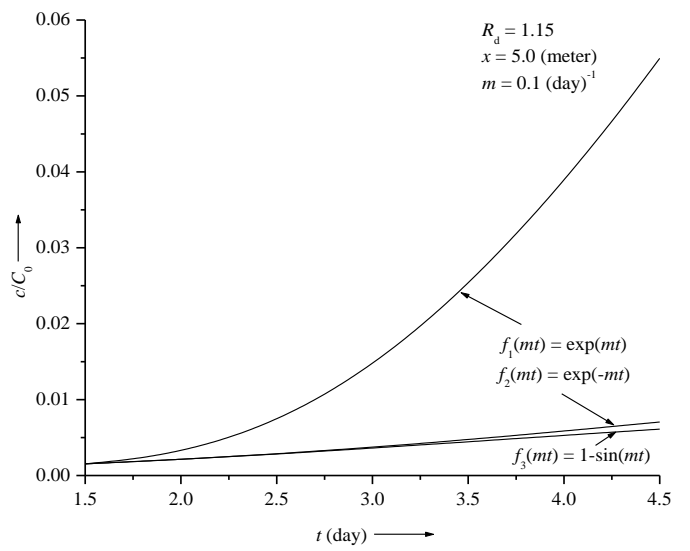

Figure 6. Comparison of the solute concentration for solution (40) at different time $t($ day $)=1.5$ to 4.5 , represented by solid curve for $f(m t)=\exp (m t)$, dashed curve for $f(m t)=\exp (-m t)$ and dotted curve for $f(m t)=1-\sin (m t)$ at particular position $x($ meter $)=5.0$.

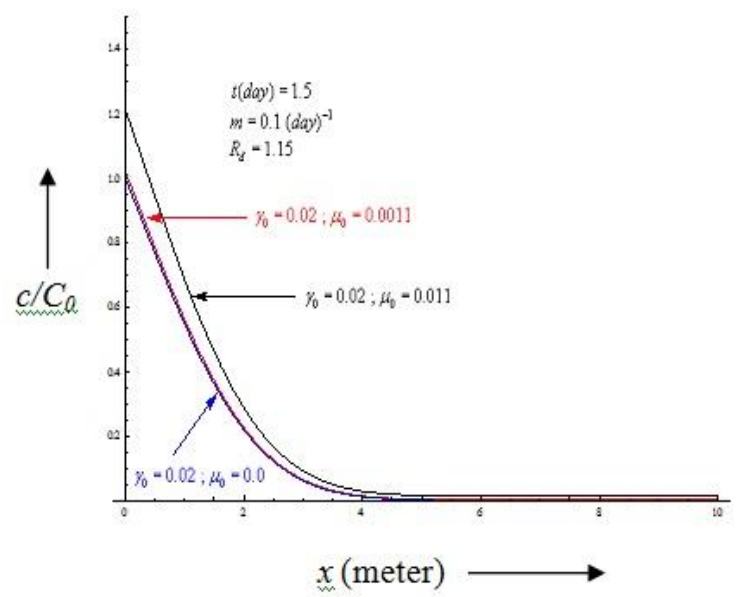

Figure 7. Effect of the production term in the presence and absence of zero-order production for distribution of solute concentration of solution (34) at one time for $f(m t)=\exp (m t)$. 